aus Sicht der Rezensentin von besonderem Wert nicht nur für die Bearbeitung der hier zusammengestellten Ursache-Wirkungshypothesen, sondern auch für die Fachbehörden in Bund und Ländern sowie für Wissenschaftler, die andere mediale und medienübergreifende Fragestellungen zu bearbeiten haben.

Auf der CD-ROM wird eine ACCESS-basierte 'Datenbank Kerndatensatz' angeboten. Durch die klar definierte Methodik und die umfassende Dokumentation der Ableitungsschritte kann der Kerndatensatz veränderten Umweltproblemen bzw. politischen Rahmenbedingungen und dem fortschreitenden Stand des Wissens angepasst werden. Insbesondere wird mit der Offenlegung der bereits überprüften aber 'verworfenen' Parameter des Kerndatensatzes in der Datenbank aus Sicht der Rezensentin sichergestellt, dass bei der notwendigen Fortschreibung des Beobachtungsprogramms Doppelarbeit vermieden werden kann.

Das im Buch und auf der CD-ROM enthaltene Glossar mit Erläuterungen zu Begriffen aus der integrierten Umweltbeobachtung ist nach Meinung der Rezensentin eine fundierte Basis für das Verständnis der Publikation und wird auch in aktuellen Veröffentlichungen als Grundlage 'für die Diskussion in der Umweltbeobachtung verwendet $(z$. B. in der Veröffentlichung des Landesamtes für Umwelt und Geologie des Freistaates Sachsen 'Umweltbeobachtung - Ziele, Strategien und Konzepte des Bundes und ausgewählter Länder', 2003).

Die Veröffentlichung zeigt insgesamt, dass sich die ökosystemare Umweltbeobachtung mit diesem Projekt in den Jahren seit dem Erscheinen der Empfehlungen des Sachverständigenrates von einer anfänglich abstrakten Vorstellung zu einem anwendbaren Instrument entwickelt hat. Das Konzept bietet den Ländern und dem Bund eine fundierte Handlungsanleitung, um die bestehenden Beobachtungsaktivitäten stärker zu koordinieren und zu bündeln, was deren Aussagequalität erhöht. Der Methodenbaukasten wurde bereits in seinem Entstehungsprozess aufwändig qualitätsgesichert. Die Begutachtungen haben ergeben, dass das Beobachtungsprogramm sowohl aus wissenschaftlicher als auch aus fachlich-administrativer Sicht grundsätzlich konsensfähig ist. Bausteine dieses auf andere Räume übertragbaren Methodenbaukastens werden neben der Erprobung und beabsichtigten Weiterführung in der Rhön bereits in den Ländern Brandenburg, SchleswigHolstein und Baden-Württemberg genutzt und stehen für weitere Anwendungen zur Verfügung.

Das Werk trägt dazu bei, die vielfältigen Ergebnisse der geleisteten Forschungsarbeit für den interessierten Leser und Anwender verstehbar und nutzbar zu machen. Durch die Verlinkung der Querverweise auf der recherchierbaren CD-ROM ist insbesondere ein schnelles Auffinden der gewünschten Information in den umfangreichen Materialien möglich. Das Werk gibt zum einen eine Fülle von Anregungen für die mit integrierter Umweltbeobachtung befassten Fachleute in Bund, Ländern und privaten Einrichtungen und alle, die sich dafür interessieren. Es vermittelt aber durch den medienübergreifenden Ansatz zugleich auch Faktenwissen über die Beobachtung aller Umweltmedien und -sektoren. Damit bietet es sich auch als Kompendium für eine breite Nutzung in Forschung und Lehre an.

Kati Mattern Umweltbundesamt, PF 3300 22, D-14191 Berlin E-mail: kati.mattern@uba.de

\title{
Buchpräsentation
}

\section{Ökotoxikologie: Umweltchemie, Toxikologie, Ökologie (Lehrbuch)}

\author{
Autor: Karl Fent \\ Verlag: Thieme (kunden.service@thieme.de). 2. überarbeitete und erweiterte Auflage 2003. 340 Seiten, 202 Abb., kartoniert. \\ $€ 49,95$. ISBN 3131099925
}

Dieses Werk

- Definiert die Ökotoxikologie als moderne multidisziplinäre Umweltwissenschaft.

- Vernetzt die aktuellen Erkenntnisse aus Umweltchemie, Toxikologie, Ökologie und sichert eine alle Aspekte umfassende Darstellung der Ökotoxikologie.

- Analysiert und untersucht die Auswirkungen der direkten und indirekten Effekte von Chemikalien auf allen biologischen Ebenen des Ökosystems einschließlich der molekularen Wirkungsmechanismen.

- Umfasst die ökotoxikologischen Untersuchungssysteme bis zur Risikoanalyse und Risikobewertung.

- Vermittelt grundlegende ökotoxikologische Konzepte.

- Entwickelt Lösungsstrategien, um Schädigungen durch die vom Menschen verursachten Veränderungen zu verhindern und Gefahren abzuwenden.

- Dokumentiert die aktuellen Umweltkatastrophen mit Schadstoffen.

Der zusammenfassende Überblick am Anfang eines jeden Kapitels dient als roter Faden. Merksätze fassen die wichtigsten Erkenntnisse zusammen. Ein ausführliches weiterführendes Literaturverzeichnis dient dem vertiefenden Studium. Zusammenfassende und zentrale Aspekte werden in Boxen dargestellt. Ein Glossar erklärt die wichtigsten Begriffe 